\title{
Creation and Use of an Electronic Health Record Reporting Database to Improve a Laboratory Test Utilization Program
}

\author{
Danielle E. Kurant ${ }^{1}$ Jason M. Baron ${ }^{1}$ Genti Strazimiri ${ }^{2}$ Kent B. Lewandrowski ${ }^{1} \quad$ Joseph W. Rudolf ${ }^{1,3}$ \\ Anand S. Dighe ${ }^{1,2}$ \\ ${ }^{1}$ Department of Pathology, Massachusetts General Hospital, Boston, \\ Massachusetts, United States \\ 2 Partners HealthCare, Boston, Massachusetts, United States \\ ${ }^{3}$ Department of Laboratory Medicine and Pathology, University of \\ Address for correspondence Anand S. Dighe, MD, PhD, Department \\ of Pathology, Massachusetts General Hospital, 55 Fruit Street, Boston, \\ MA 02114-2696, United States (e-mail: asdighe@partners.org).
} Minnesota Medical School, Minneapolis, Minnesota, United States

Appl Clin Inform 2018;9:519-527.

\section{Abstract}

Keywords

- data analysis

- laboratory information systems

- pathology

- monitoring and surveillance

- order entry
Objectives Laboratory-based utilization management programs typically rely primarily on data derived from the laboratory information system to analyze testing volumes for trends and utilization concerns. We wished to examine the ability of an electronic health record (EHR) laboratory orders database to improve a laboratory utilization program.

Methods We obtained a daily file from our EHR containing data related to laboratory test ordering. We then used an automated process to import this file into a database to facilitate self-service queries and analysis.

Results The EHR laboratory orders database has proven to be an important addition to our utilization management program. We provide three representative examples of how the EHR laboratory orders database has been used to address common utilization issues. We demonstrate that analysis of EHR laboratory orders data has been able to provide unique insights that cannot be obtained by review of laboratory information system data alone. Further, we provide recommendations on key EHR data fields of importance to laboratory utilization efforts.

Conclusion We demonstrate that an EHR laboratory orders database may be a useful tool in the monitoring and optimization of laboratory testing. We recommend that health care systems develop and maintain a database of EHR laboratory orders data and integrate this data with their laboratory utilization programs.

\section{Background and Significance}

Cost, quality, outcomes, and patient safety concerns continue to motivate efforts to improve the utilization of health care services, including the laboratory. Health care providers have been estimated to influence up to $80 \%$ of the costs of health care, ${ }^{1}$ and it is widely believed that laboratory data are a major driver of medical decision making. ${ }^{2}$ In addition, stu- dies have shown that the usage of medical services, including procedures and laboratory tests, varies greatly based on region and geography., ${ }^{3,4}$ This high level of variability suggests inappropriate utilization, including both over- and underutilization of laboratory testing. ${ }^{5}$ With the limited availability of medical resources, the need for judicious utilization of laboratory tests becomes essential. received

March 28, 2018

accepted after revision

May 26, 2018 (c) 2018 Georg Thieme Verlag KG Stuttgart · New York
DOI https://doi.org/

$10.1055 / \mathrm{s}-0038-1666843$. ISSN 1869-0327. 
Health care systems have responded to the need to closely manage laboratory testing with the formation of laboratory utilization management programs designed to monitor new and existing test usage. ${ }^{6,7}$ Data required for these efforts typically consists of reports from the laboratory information system (LIS) to provide test level information including volume, result values, and provider data for the tests to be monitored. However, while LIS data are important in monitoring test usage, in our experience the information collected in LIS reports is often insufficient for comprehensive and efficient utilization management. ${ }^{8}$

The inability of LIS data alone to provide insight into many utilization issues appears to be largely due to the complexities introduced into the laboratory test ordering process with the increased prevalence and capabilities of the electronic health record (EHR). ${ }^{9,10}$ An increasing percentage of health care systems worldwide now utilize an EHR that typically includes many different pathways for laboratory ordering by providers, including selecting from lists, searching, and using predefined order sets and panels. ${ }^{11-13}$

Well-designed EHR systems provide a platform to streamline workflow, standardize laboratory ordering, promote adherence to guidelines, and deliver decision support alerts. ${ }^{14-16}$ With several hundred to thousands of tests on a typical laboratory menu, search functionality is found in most EHR implementations that permits providers to rapidly search for a laboratory order in a given list of laboratory tests. With search, clinicians enter search terms and the system returns a list of matching tests. In addition to searching for laboratory tests, EHRs typically allow providers to create personalized preference lists with tests they find particularly helpful or order most frequently. Further, standardized order sets provided in most EHR implementations offer an opportunity for the system to guide order selection by clinicians. ${ }^{17-20}$ Order sets are groups of orders that are frequently ordered together. For example, an anemia order set may include a complete blood count, ferritin, total iron binding capacity, serum folate, vitamin B12, and/or other related tests.

It is well established that timely access to LIS data is useful to inform and improve test utilization. ${ }^{8}$ However, to our knowledge no reports have examined the usage of an EHR reporting database to enhance a laboratory utilization program. Herein, we describe the construction of an EHR laboratory orders database that we have used to generate targeted analyses to advance a utilization management program.

\section{Objectives}

The aims of this study were (1) to develop a near real-time EHR laboratory orders database with data relevant to laboratory utilization, (2) to examine the ability of the EHR laboratory orders database to improve an established laboratory utilization program, and (3) to document the key EHR fields with the highest value to laboratory utilization management.

\section{Methods}

\section{Setting}

These initiatives were performed at the Massachusetts General Hospital (MGH), a 999-bed tertiary care teaching hospital in Boston, Massachusetts, United States. The MGH is a founding member of Partners HealthCare, a multi-institutional, collaborative, not-for-profit health care system. These projects were completed as quality improvement initiatives and, as such, were not formally reviewed by the institutional review board, per protocol.

The MGH Clinical Laboratories produce over 10 million test results per year with an EHR orderable test menu of over 1,500 tests. Laboratory test utilization is managed primarily by faculty and staff within the Department of Pathology, which contains multiple interdisciplinary committees involved in test addition and removal, utilization, and monitoring of utilization initiative effectiveness. ${ }^{6}$ The Core Laboratory, under the umbrella of the Department of Pathology, is also responsible for the budget for laboratory testing that is sent outside the system to be performed by reference laboratories.

The Partners HealthCare system, including the MGH, uses Epic (Epic Clinical Systems, Verona, Wisconsin, United States) as its EHR system. The Epic EHR is utilized in all clinical areas including inpatient, outpatient, and emergency department settings. The MGH Clinical Laboratories also use the Sunquest LIS (Sunquest Information Systems, Tucson, Arizona, United States).

\section{EHR Orders Database Creation}

We modified an existing Structured Query Language Server (Microsoft, Redmond, Washington, United States) database to accommodate the new EHR test ordering data. We had previously used this database to import and store LIS data, including all laboratory test results. In collaboration with our health system's Epic reporting team, we developed an EHR report containing fields pertinent to all laboratory orders that were updated (e.g., ordered, released, resulted, or cancelled) the prior day. Fields of interest to our utilization program are captured in the EHR report, examples of which are shown in -Table 1. Recommendations and comments on the content of the EHR report are presented in the "Discussion" section.

The EHR laboratory orders report is generated daily via Epic's "Clarity" reporting database and sent as a delimiter separated file to a shared file area. The EHR laboratory orders database was configured to automatically import these files using a structured process to parse and store the EHR data. All EHR data are stored in a single table. Typical daily EHR report files are 60 to 70 megabytes in size, and contain 140 to 160,000 rows of data. The import process incorporates a variety of automated quality assurance functions including file validation, data validation, and error notification. The process automatically archives the original delimited report after importing it. Users generally access the EHR orders database via Open Database Connectivity (ODBC) and Microsoft Access (Microsoft). Data analysis is performed in Microsoft Excel, Microsoft Access, R, or Python. 
Table 1 Selected key data fields imported from an EHR report into the EHR orders database

\begin{tabular}{|c|c|c|c|}
\hline Data field & Information type & $\begin{array}{l}\text { Availability } \\
\text { in LIS }\end{array}$ & Comment \\
\hline Enterprise medical record number & Patient information & No & $\begin{array}{l}\text { Global medical record number binding medical record } \\
\text { numbers at individual sites }\end{array}$ \\
\hline Local medical record number (site 1 ) & Patient information & Yes & Medical record number for principal site \\
\hline Local medical record number (site 2 ) & Patient information & No & Medical record number (if present) for sister academic site \\
\hline Encounter number & Patient information & Yes & $\begin{array}{l}\text { Unique identifier for patient encounter during which } \\
\text { laboratory was ordered }\end{array}$ \\
\hline Ordering department identifier & Provider & Yes & Department of ordering provider \\
\hline Authorizing provider identifier & Provider & No & Department of authorizing provider \\
\hline Ordering provider identifier & Provider & Yes & Numeric provider identification number \\
\hline Ordering provider location & Provider & Yes & Provider's practice location \\
\hline EHR order number & Order information & Yes & $\begin{array}{l}\text { Provides link to LIS result, as EHR order number is stored } \\
\text { with the result within the LIS }\end{array}$ \\
\hline Order date/Time & Order information & Yes & Date and time of order \\
\hline Order source & Order information & No & Application within EHR where order was placed \\
\hline Procedure code & Order information & No & Laboratory order code in EHR \\
\hline Display name & Order information & No & Laboratory order display name in EHR \\
\hline Cancellation reason & Order information & Yes & For cancelled tests, reason for cancellation \\
\hline Priority information & Order information & Yes & Routine or STAT \\
\hline Expected date & Order information & No & Date laboratory is expected to be collected \\
\hline Expiration date & Order information & No & Date when order will expire \\
\hline Release date/Time & Order information & Yes & $\begin{array}{l}\text { Date/time that laboratory order was sent to laboratory } \\
\text { information system }\end{array}$ \\
\hline Accession number & Order information & Yes & $\begin{array}{l}\text { Accession number within LIS, if laboratory is in process } \\
\text { or resulted }\end{array}$ \\
\hline Provider order entry comments & Patient-specific order details & No & $\begin{array}{l}\text { Comments made by the provider in the EHR about this order } \\
\text { during the ordering process }\end{array}$ \\
\hline Order entry questions & Patient-specific order details & No & Prompts asked of provider when ordering test \\
\hline Order set identifier & Order origination information & No & Order set from which order was selected from \\
\hline Order set name & Order origination information & No & Name of order set displayed to provider in EHR \\
\hline Laboratory preference list name & Order origination information & No & Preference list where order was placed \\
\hline Resulting laboratory identifier & Order origination information & No & $\begin{array}{l}\text { Resulting laboratory that was populated or selected } \\
\text { during order entry }\end{array}$ \\
\hline Resulting laboratory name & Order origination information & No & Name of laboratory where testing will be performed \\
\hline
\end{tabular}

Abbreviations: EHR, electronic health record; LIS, laboratory information system.

Note: The availability or lack of availability of these EHR fields in the laboratory information system is noted.

\section{Results}

We have utilized the EHR orders database as a central part of our laboratory utilization program. Below, we provide three representative examples demonstrating how the EHR orders database has been integrated into our program. The ability to have detailed information on each EHR laboratory order, including the providers involved, the EHR application module, and point of origin of the order, has allowed us to rapidly respond to shifts in laboratory ordering and to make targeted changes to the EHR to respond to the identified utilization issues. We provide examples below in the areas of volume monitoring, search optimization, and miscellaneous test monitoring.

\section{Volume Monitoring}

One of the key tasks for utilization management programs is to monitor for unexpected changes in test volumes. Unex- pected increases in individual tests may reflect practice changes, provider hiring, EHR menu changes, new EHR order set usage, or EHR errors. These changes are important to track since, in addition to impacting the diagnostic efficiency of laboratory workups, they may also directly impact the laboratory operating budget. In the case of high-cost reference laboratory testing, even a small shift in test ordering can quickly have a significant budgetary impact.

With the use of a routine LIS monitoring report that compares the most recent weekly and monthly test volumes of all tests to historical test result volumes, a nearly 10 -fold increase in red blood cell (RBC) folate ordering was noted over a period of several weeks ( - Fig. 1). This was concerning as the serum folate test is typically preferred over RBC folate for evaluating patients with suspected folate deficiency. ${ }^{21}$ Further, while serum folate is an inexpensive test performed 24 hours a day with rapid turnaround time in our laboratory, 


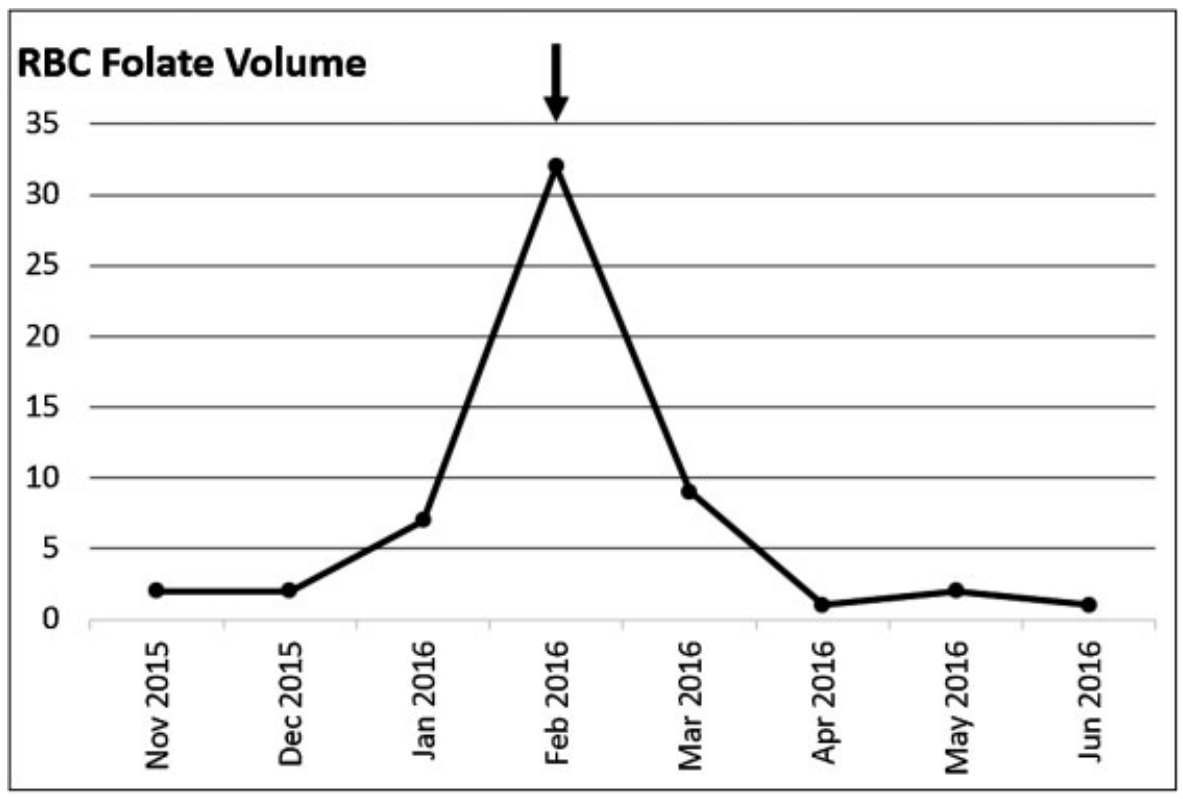

Fig. 1 Red blood cell (RBC) folate monthly volumes. The arrow indicates the month where the anemia order set containing RBC folate was updated to include serum folate in the place of RBC folate.

$\mathrm{RBC}$ folate is an expensive test performed at a reference laboratory with a much longer turnaround time. Moreover, the Choosing Wisely Collaborative, along with the American Society for Clinical Pathology, has recently identified RBC folate as a test that should rarely be ordered. ${ }^{22}$

Our LIS monitoring report, although capable of identifying the volumes and providers that ordered the testing, was unable to provide the context of the ordering process, so the LIS report was of limited value in determining the reason behind the increase in RBC folate ordering. Thus, we examined our EHR orders database to analyze the EHR orders and attempt to determine the root cause for the increase in RBC folate ordering.

An understanding as to how the orders originated in the EHR is important to be able to design an effective intervention. Key fields in our EHR orders database (see the "Discussion" section for listing of database fields) allow us to determine if the order originated from a provider selecting the order from the full list of all available tests, a specialty preference list (e.g., Pediatrics test list), a provider's personal preference list (i.e., their "favorites"), or an order set. In this case, most RBC folate requests were shown to be ordered from a single "Anemia" order set ( $>90 \%$ of total orders, data not shown). With knowledge of the origin of the orders, we contacted the clinical group overseeing the anemia order set to request removal of the RBC folate test and its replacement with serum folate. Following the intervention, the frequency of RBC folate orders quickly returned to its prior baseline (-Fig. 1).

\section{Menu/Search Optimization}

A key consideration for every health care system is which tests are available on the laboratory testing menu (i.e., the laboratory formulary). ${ }^{23}$ The choices presented to the user when searching for a laboratory test are important determinants for what eventually will be ordered by the clinician. Naming conventions, synonyms, and sort order may all influence the provider's eventual choice of test. ${ }^{24}$ Inappropriate provider test selections may have significant downstream workflow impacts on the laboratory if the tests that are overutilized are highly manual compared with the more appropriate testing.

For patient workups for monoclonal gammopathies, a typical screening test is the serum protein electrophoresis (SPEP) panel. In our institution, we utilize an institutional reflex protocol when a SPEP panel is ordered. We first perform a SPEP, serum immunoglobulins, and total protein testing and the results of these tests are then reviewed by a laboratory medical director. At that point, based on prior history, immunoglobulin levels, SPEP findings, and EHR review, the director may determine the need to perform serum immunofixation testing to further evaluate the sample and/or further characterize any identified $\mathrm{M}$ components.

When we migrated our institution to a new EHR (Epic), we included two tests related to monoclonal gammopathy evaluation on the EHR laboratory menu, namely, "SPEP panel" and "SPEP panel with immunofixation." When the former test was ordered, the laboratory would perform the reflex protocol outlined above and only perform immunofixations when indicated by the algorithm. In contrast, when "SPEP panel with immunofixation" was ordered, the laboratory would always perform both a SPEP and an immunofixation on the sample.

Following implementation of the new EHR, the immunology section of our laboratory reported a significant increase in immunofixation testing. This was substantiated by our LIS monitoring report demonstrating a steady increase in serum immunofixation orders (-Fig. 2). In our prior order entry systems, before implementation of the new EHR, the "SPEP panel with immunofixation" test was ordered at a much lower frequency. Detailed review of LIS volume reports for the two tests did not demonstrate any clusters of providers, locations, or other clues to the basis for the increased ordering of serum immunofixation. 


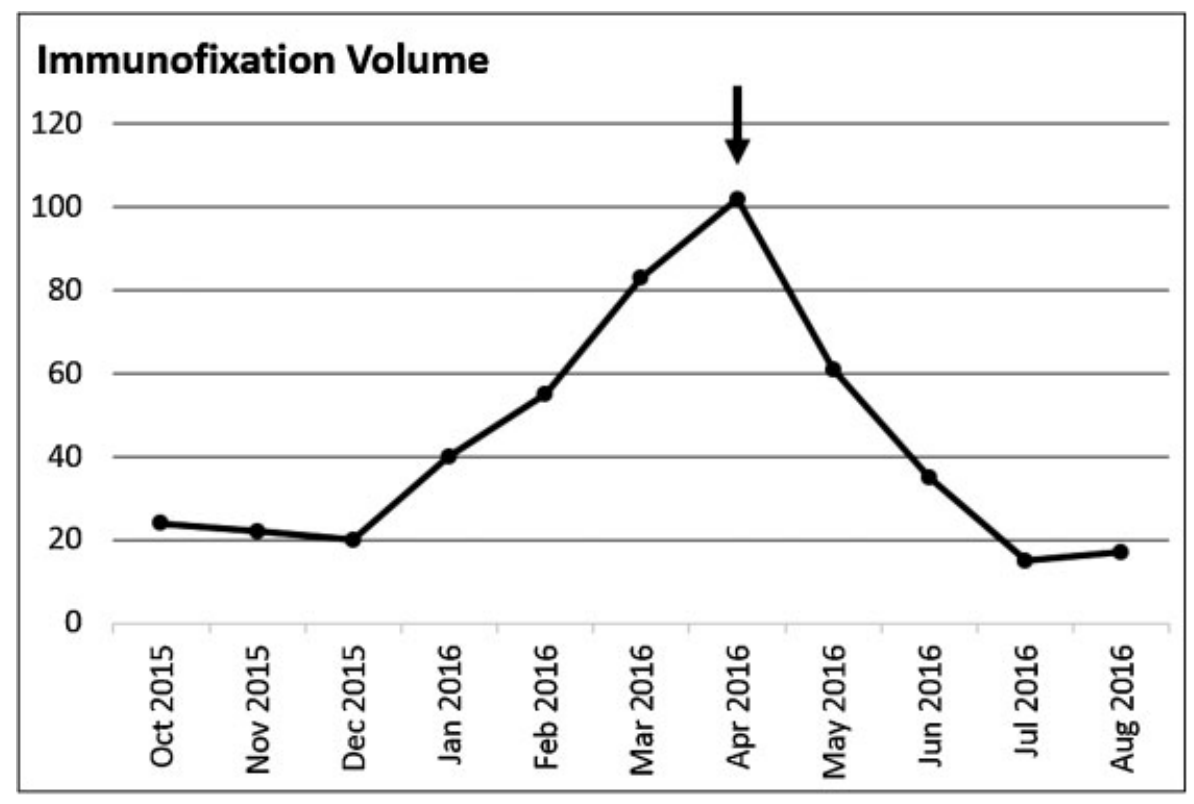

Fig. 2 Serum immunofixation monthly volumes. The arrow indicates where the electronic health record (EHR) order for serum protein electrophoresis (SPEP) with immunofixation was removed from the EHR menu.

Thus, to provide further insight to how the two tests were being ordered, we reviewed our EHR orders database. In contrast to the RBC folate example above, review of the EHR orders database for these tests indicated that most "SPEP with immunofixation" tests were not originating from order sets or provider favorites. Rather, most SPEP with immunofixation orders were selected following a search of the full outpatient test menu ( $72 \%$ of total SPEP with immunofixation orders, data not shown). This information that was obtained from the EHR orders report and not available in the LIS was important in planning our intervention.

In many situations, limiting EHR options is an efficient approach to managing test utilization. ${ }^{23}$ In this case, as determined by the EHR data, a key decision point occurred when clinicians performed a search of the outpatient test menu and they were presented with two options "SPEP panel" and "SPEP panel with immunofixation." At this point in the ordering process, many users appear to have selected the choice that appeared to be more comprehensive ("SPEP panel with immunofixation"), not appreciating that the standard "SPEP panel" has an associated reflex protocol that would order the immunofixation when indicated.

After conferring with providers, we confirmed that we could eliminate the SPEP with immunofixation test from the EHR menu. One consideration with removal of a test from the menu is that providers may have added that test to their personal laboratory preference list (their "favorites"). With our EHR orders database, we also noted that a significant number of the orders originated from provider personal preference lists (22\% of total SPEP with immunofixation orders, data not shown). To transition these users to using the standard SPEP panel, we requested a change to over 50 personal preference lists involving substitution of the more desirable SPEP panel for the less desirable test, SPEP panel with immunofixation. Following these interventions, the serum immunofixation order frequency returned to its prior baseline (-Fig. 2).

\section{Miscellaneous Test Monitoring}

The EHR laboratory testing menu for our hospital contains over 1,500 individual tests. These tests were selected on the basis of test volumes, clinical assessment of their utility, and certain other workflow considerations. However, as a matter of practicality, infrequently needed tests, including many esoteric and highly specialized genetic and microbiologic tests used to diagnose rare diseases, were excluded from the test menu. Indeed, there were likely several thousand tests that we could have put on our test menu, most of which would be rarely (e.g., several times per year or less) if ever ordered. We chose not to include these tests on the EHR testing menu for several reasons. First, many of these tests should not be ordered except in highly selected conditions and adding them to the general test menu could lead to their use in situations where they are not indicated, resulting in inappropriate utilization and increased cost. In addition, adding each test to the EHR menu requires mapping, testing, and maintenance to ensure the orders can be electronically interfaced to the LIS and that the results will be faithfully transmitted from the LIS to the EHR.

There are several options for managing miscellaneous test requests in EHR implementations. One solution is to not permit miscellaneous test ordering in the EHR. This option generally requires providers to fill out a paper requisition form when requesting miscellaneous tests. While paper requisitions could presumably limit the number of test orders by making it inconvenient to order miscellaneous tests, this option presents several problems. First, paper requisitions make it difficult to track the orders being placed and do not permit the EHR and LIS to produce barcoded labels for the orders to facilitate "closed loop" order to result tracking. In addition, paper requisitions also introduce 
multiple opportunities for transcription error and add labor to each order as these test requests must be ordered in the LIS upon reaching the laboratory.

To prevent the challenges associated with paper requisitions, we chose to offer a "Miscellaneous laboratory test" order in our EHR that can be utilized to place an electronic request for "write-in" tests. When ordering the Miscellaneous test request, the provider is prompted to provide the name of the tests they are ordering as well as any other details they may be aware of such as the tube type or the preferred performing laboratory. Having the "Miscellaneous laboratory test" as an orderable test within our EHR menu permits an electronic order to be sent to the LIS and a barcoded label to be generated to facilitate sample collection and tracking.

The use of an EHR-orderable "Miscellaneous test" strategy requires a commitment to monitoring these tests. If used to circumvent the standard test menu, the inappropriate use of the "Miscellaneous test" order can lead to incorrect tube types, insufficient specimen, and delays in specimen processing. We determined that the ability to track these orders was essential to our continued efforts to optimize test utilization.

Having an electronic order permits the utilization of our combined EHR orders and LIS results database to analyze each miscellaneous order request and determine if it is appropriate. In our utilization database, we employ the EHR order number to link the EHR order with the LIS result, as the EHR order number is sent to the LIS and associated with the test result. At our institution in 2017 approximately $0.4 \%$ of orders placed ( 40 of 9,500 laboratory orders placed per day) were miscellaneous test requests.

With the use of the utilization database to analyze both the miscellaneous test orders being placed as well as the tests that were eventually resulted from these orders, we have been able to use the miscellaneous test order and its monitoring to continuously improve laboratory quality. Using the EHR laboratory orders database, we analyze the context of each miscellaneous test order to understand the EHR module that the order originated, the responses in the EHR to required questions, and examine the other orders that were ordered during the same ordering session.
The analysis of each miscellaneous test order has several possible outcomes as outlined in -Table 2. Outcomes of the analysis include the following: (1) If a clinically useful test that is not on the current EHR menu is being ordered frequently as a miscellaneous test, it would likely be of value to add the test to the EHR test menu. (2) Tests that are uncommon but deemed appropriate in the ordering context of the patient can remain as miscellaneous tests (i.e., remain off the structured test menu). (3) When tests that are currently on the existing EHR test menu were ordered via the miscellaneous order code instead of the structured order, one possibility is that the clinician was unable to find the test after searching the EHR test menu. ${ }^{24,25}$ In these cases, reviewing the synonyms defined in the EHR for the test, verifying the inclusion of the test on relevant facility and specialty preference lists, and/or educating clinicians would be appropriate next steps. In this scenario, we also analyze the EHR laboratory orders database for those providers that have correctly found the structured order of interest to understand if order availability issues, including the orders' presence or absence on order sets, facility lists, or specialty preference lists, may contribute to a given provider's ability to find the correct test. (4) In some circumstances, orders are placed for miscellaneous tests that are unhelpful, outdated, or inappropriate for the clinical context. In this case, an appropriate action would be to educate clinicians rather than make any changes to the EHR test menu.

Thus, by capturing detailed information in our EHR database regarding miscellaneous test requests we have been able to continuously adapt our test menu according to the needs of our clinicians as well as monitor the appropriateness of the orders being placed. Importantly, much of the key information that is used to make decisions during the analysis of miscellaneous test orders is derived solely from the EHR orders database and is not found in the LIS.

\section{Discussion}

As the cost of health care continues to escalate, the need for effective utilization management programs becomes ever more important. ${ }^{26}$ The increasingly ubiquitous role of the

Table 2 Possible outcomes to EHR miscellaneous test monitoring analysis

\begin{tabular}{|l|l|l|}
\hline Conclusion of EHR order review & Next steps & Comment \\
\hline $\begin{array}{l}\text { High volume test that is not currently } \\
\text { available to be ordered in the EHR and will } \\
\text { be used in future }\end{array}$ & $\begin{array}{l}\text { Add test to the EHR facility list for future } \\
\text { ordering }\end{array}$ & Add to EHR facility list \\
\hline $\begin{array}{l}\text { "One time" or high cost esoteric test not } \\
\text { currently available to order in the EHR }\end{array}$ & $\begin{array}{l}\text { Appropriate as miscellaneous. Do not } \\
\text { add to EHR facility list }\end{array}$ & No further action needed \\
\hline $\begin{array}{l}\text { Test already built in the EHR and on the } \\
\text { current EHR menu }\end{array}$ & $\begin{array}{l}\text { Review EHR synonyms, test display } \\
\text { name, order sets, and preference lists to } \\
\text { ensure test can be easily found with } \\
\text { search and that test is on appropriate } \\
\text { lists and order sets. Make EHR changes } \\
\text { as needed }\end{array}$ & $\begin{array}{l}\text { Educate clinician if recurrent issue } \\
\text { and no EHR systematic issue is found }\end{array}$ \\
\hline $\begin{array}{l}\text { Test not on menu and is not appropriate for } \\
\text { clinical context }\end{array}$ & No change to EHR menu & Educate clinician \\
\hline
\end{tabular}

Abbreviation: EHR, electronic health record. 
EHR in facilitating electronic laboratory test ordering has made an understanding of EHR workflows and ordering essential to clinical laboratory test utilization management programs. In addition, while the EHR contains literally thousands of data points on every patient, there is a relatively constrained subset of data that is useful for understanding the details and context of an individual laboratory order. In this report, we identified a subset of information that could be readily extracted from the EHR and then examined whether this information provides value to our laboratory utilization management program. We demonstrate that knowledge of the EHR details of the order provides valuable insights into the test ordering process and may be useful in the formulation of EHR-based solutions to a given utilization issue. We recommend that all laboratories develop and maintain near real-time data from the EHR to assist with utilization management initiatives.

The approach described here should be applicable to most health care settings where the EHR is a dominant method for laboratory test ordering. With the increased use of the EHR throughout health care systems, more and more systems have now implemented electronic laboratory ordering within the EHR. ${ }^{9}$ In addition, most EHR systems have been designed with reporting needs considered and many types of reports can typically be generated from the EHR. It is important that these reports be generated in a near realtime manner (e.g., next day) since laboratory utilization patterns can change rapidly. This may be especially the case in the event of an EHR error in mapping or menu updating that could inadvertently have a significant and immediate impact on laboratory test ordering.

The aggregation and importing of daily EHR reports into a queryable EHR orders database offers numerous advantages compared with the individual EHR reports alone. First, it allows custom queries to be written whenever a utilization issue arises and to be able to run these queries across any time period of interest to observe trends and trajectories of testing. In addition, the EHR orders database rapidly generates a historical record of EHR activity that can serve as a baseline for utilization monitoring efforts. This baseline allows the determination of volume trends and the ability to observe shifts in the process of how tests are ordered.

Having these data accessible to a wide range of individuals limits the number of custom EHR reports that need to be requested, decreasing the demands on resource-constrained EHR reporting teams and reducing the time required for analysis. Thus, in addition to data extraction and storage processes it is also necessary for organizations to develop user-friendly interfaces for laboratory staff to visualize and interact with the data to facilitate end-user engagement. This interaction can take a variety of forms, including direct access to the database via ODBC connections, visualizations of the database built into programs such as spreadsheet software or programming languages like $\mathrm{R}$ or Python, or even dashboards directly accessing the EHR orders database. The means of user interaction with the database depends on the needs and experience of the end user, and therefore should be customized to suit the needs of each individual organization. We have created our database in such a way that it can be securely and directly accessed by data scientists via direct connections, but also have developed simpler interfaces, including Microsoft Access reports, for laboratory directors and other laboratory staff to access the information for utilization and quality efforts such as this study.

Our costs for the EHR database creation were modest, as we simply added a single table to an existing database and modified an existing procedure to extract and store the EHR report data in the database. As opposed to highly clinical EHR data, such as medications and problem list information, which tend to be complex, the EHR orders data does not require high levels of processing or analysis to make the data useful for utilization management. We primarily use simple Microsoft Access-based queries via the EHR reporting database for our utilization initiatives.

Awareness of the EHR details of test ordering, including the frequency, involved providers, and context, allows the clinical laboratory to identify trends in test usage, consider additions and removals to the test menu, consider modifications to existing order sets, and identify targets for more advanced decision support. In our experience, certain EHR fields have been demonstrated to be useful to include in reports used for test utilization monitoring. These fields fall into three major groupings: patient data, provider data, and order data. - Table 1 provides a listing of some of the key fields included in our EHR orders report along with some comments regarding the individual fields. It further delineates which of the EHR order report fields could potentially be obtained from a LIS report.

The EHR provider data elements that have proven to be useful for laboratory utilization efforts include the entire team who may be involved in the order. A limitation of LIS provider data are that typically only a single provider is electronically sent to the LIS in the electronic ordering message. In a training environment, however, there can be several providers involved in the decision to order a laboratory test (e.g., attending physician, fellow, resident, nurse practitioner, medical assistant, etc.). Knowledge of the relevant provider is essential to targeting solutions to many utilization and quality issues. Our EHR orders report captures both the ordering provider as well as the provider responsible for follow-up and billing.

The details of the order that have been shown to be especially useful to laboratory utilization efforts include the ordering location and "order origination" information. In particular, the details of order origination, including the EHR module, encounter type, preference list, and order set, are not typically sent to the LIS and are thus unavailable to laboratories when doing utilization analysis based on LIS reports alone. In the EHR there are many potential modules and pathways that a given laboratory order can originate from, including orders selected after searching the full facility list of tests, orders selected from personal preference lists, orders from departmental preference lists, and orders selected from within an order set. For each laboratory order, the specific originating location is specified in our EHR orders database. Knowledge of the origin of the order within the 
EHR provides our utilization management team with a clear target for interventions that cannot be determined from simple LIS volume data. In many cases based on this EHR orders data, we have dramatically improved utilization practices by editing a single order set or departmental preference list. Without this knowledge of where the inappropriate orders were originating, we would not have been able to customize our solutions to fit the issue or would have had to spend time reviewing EHR patient charts to attempt to determine the context of the orders.

The inclusion of additional EHR information in the database, including patient medications, problem lists, and other clinical data that may impact test ordering and resulting, is a future direction of our work. Ready access to these additional EHR data elements may improve the ability of the laboratory to perform institution-specific reflex protocols, provide clinically relevant interpretive comments, and improve the efficiency and quality of laboratory interpretive services.

\section{Conclusion}

Herein, we have observed the utility of an EHR orders database to provide insight into a variety of utilization challenges. EHR orders data, particularly when combined with LIS data, can provide novel insights and identify ordering patterns by clinician, department, EHR application module, EHR preference list, EHR order set, patient encounter type, and patient diagnosis. We demonstrate that incorporating this information into a database framework facilitates analysis and utilization optimization.

\section{Clinical Relevance Statement}

This article demonstrates the value of aggregated electronic health record (EHR) data to improve a laboratory test utilization program. While the EHR contains thousands of distinct data fields, a small subset of EHR laboratory orders data was identified in the study and shown to be valuable for test utilization initiatives. As the EHR increasingly becomes the primary means of laboratory ordering, the context and ordering pathways within the EHR will become increasingly important for health care systems to be able to analyze and monitor.

\section{Multiple Choice Questions}

1. Which of these data fields are often lacking in laboratory information system reports?

a. Laboratory test name

b. Laboratory test cost

c. EHR order set from which the test was ordered

d. Laboratory order number

Correct Answer: The correct answer is option c. The laboratory order number, test name, and test cost are generally associated with the test code in the laboratory information system (LIS). The order origination information, such as which order set the order originated, are not sent to the LIS. Order origination information is valuable information for utilization management programs to be able to target interventions within the EHR.

2. What is the value of a laboratory order EHR database versus individual daily EHR reports of laboratory ordering activity?

a. Lower cost to create and maintain a database than generating reports

b. More data fields can be extracted and stored in a database than in a daily report

c. EHR orders database information cannot easily be combined with other data whereas reports can be easily combined

d. Longitudinal queries and analysis can be performed on an orders database to assess for trends.

Correct Answer: The correct answer is option d. Although database construction and maintenance may incur costs not associated with creating simple reports, the costs can be modest if a constrained subset of data is stored. A key advantage of having laboratory order information in a database is that longitudinal analysis can be performed to identify trends and ordering patterns. Moreover, databases typically provide a simple interface to link data stored in one database with other key data sources. In the case of laboratory utilization management, we routinely associate EHR orders data with laboratory results data to provide a useful window into our combined EHR and laboratory operations.

\section{Protection of Human and Animal Subjects}

This study was performed in compliance with the World Medical Association Declaration of Helsinki on Ethical Principles for Medical Research Involving Human Subjects.

Conflict of Interest

None.

\section{References}

1 Berndtson K. Managers and physicians come head to head over cost control. Healthc Financ Manage 1986;40(09):23-24

2 Becich MJ. Information management: moving from test results to clinical information. Clin Leadersh Manag Rev 2000;14(06): 296-300

3 Gornick M. Medicare patients: geographic differences in hospital discharge rates and multiple stays. Soc Secur Bull 1977;40(06): 22-41

4 Welch WP, Miller ME, Welch HG, Fisher ES, Wennberg JE. Geographic variation in expenditures for physicians' services in the United States. N Engl J Med 1993;328(09):621-627

5 Zhi M, Ding EL, Theisen-Toupal J, Whelan J, Arnaout R. The landscape of inappropriate laboratory testing: a 15-year metaanalysis. PLoS One 2013;8(11):e78962

6 Kim JY, Dzik WH, Dighe AS, Lewandrowski KB. Utilization management in a large urban academic medical center: a 10-year experience. Am J Clin Pathol 2011;135(01):108-118

7 Huck A, Lewandrowski K. Utilization management in the clinical laboratory: an introduction and overview of the literature. Clin Chim Acta 2014;427:111-117

8 Baron JM, Dighe AS. The role of informatics and decision support in utilization management. Clin Chim Acta 2014;427:196-201 
9 Adler-Milstein J, Holmgren AJ, Kralovec P, Worzala C, Searcy T, Patel V. Electronic health record adoption in US hospitals: the emergence of a digital "advanced use" divide. J Am Med Inform Assoc 2017;24(06):1142-1148

10 Adler-Milstein J, DesRoches CM, Kralovec P, et al. Electronic health record adoption in US hospitals: progress continues, but challenges persist. Health Aff (Millwood) 2015;34(12):2174-2180

11 Gold M, McLaughlin C. Assessing HITECH implementation and lessons: 5 years later. Milbank Q 2016;94(03):654-687

12 Guo U, Chen L, Mehta PH. Electronic health record innovations: Helping physicians - one less click at a time. Health Inf Manag 2017;46(03):140-144

13 Lammers EJ, McLaughlin CG. Meaningful use of electronic health records and medicare expenditures: evidence from a panel data analysis of U.S. health care markets, 2010-2013. Health Serv Res 2017;52(04):1364-1386

14 Georgiou A, Vecellio E, Toouli G, et al. Monitoring the impact of the electronic medical record on the quality of laboratory test ordering practices. Stud Health Technol Inform 2013;188:33-38

15 Georgiou A, Morse W, Timmins W, Ray S, Westbrook JI. The use of performance metrics to monitor the impact of CPOE on pathology laboratory services. Stud Health Technol Inform 2008;136:291-296

16 Petrides AK, Bixho I, Goonan EM, et al. The benefits and challenges of an interfaced electronic health record and laboratory information system: effects on laboratory processes. Arch Pathol Lab Med 2017;141(03):410-417

17 Mekhjian H, Saltz J, Rogers P, Kamal J. Impact of CPOE order sets on lab orders. AMIA Annu Symp Proc 2003:931
18 Wright A, Sittig DF, Carpenter JD, Krall MA, Pang JE, Middleton B. Order sets in computerized physician order entry systems: an analysis of seven sites. AMIA Annu Symp Proc 2010; 2010:892-896

19 Olson J, Hollenbeak C, Donaldson K, Abendroth T, Castellani W. Default settings of computerized physician order entry system order sets drive ordering habits. J Pathol Inform 2015;6:16

20 Zhang Y, Levin JE, Padman R. Data-driven order set generation and evaluation in the pediatric environment. AMIA Annu Symp Proc 2012;2012:1469-1478

21 Farrell CJ, Kirsch SH, Herrmann M. Red cell or serum folate: what to do in clinical practice? Clin Chem Lab Med 2013;51 (03):555-569

22 Choosing Wisely Campaign. Available at: http://www.choosingwisely.org/societies/american-society-for-clinical-pathology/. Accessed May 7, 2018

23 Jackson BR. Laboratory formularies. Clin Chim Acta 2014; 427:151-153

24 Grisson R, Kim JY, Brodsky V, et al. A novel class of laboratory middleware. Promoting information flow and improving computerized provider order entry. Am J Clin Pathol 2010;133(06): 860-869

25 Blechner M, Kish J, Chadaga V, Dighe AS. Analysis of search in an online clinical laboratory manual. Am J Clin Pathol 2006;126(02): 208-214

26 Keehan SP, Cuckler GA, Sisko AM, et al. National health expenditure projections, 2014-24: spending growth faster than recent trends. Health Aff (Millwood) 2015;34(08):1407-1417 